\title{
[Fe XIV] and [Fe XI] reveal the forward shock in SNR 1E 0102.2-7219
}

\author{
Frédéric P. A. Vogt ${ }^{1, \star}$, Ivo R. Seitenzahl ${ }^{2,3,4}$, Michael A. Dopita ${ }^{2}$, and Parviz Ghavamian ${ }^{5}$ \\ ${ }^{1}$ European Southern Observatory, Av. Alonso de Córdova 3107, 7630355 Vitacura, Santiago, Chile \\ e-mail: frederic.vogt@alumni.anu.edu.au \\ 2 Research School of Astronomy and Astrophysics, Australian National University, 0200 Canberra, Australia \\ 3 ARC Centre for All-sky Astrophysics (CAASTRO), 2006 NSW, Australia \\ 4 School of Physical, Environmental and Mathematical Sciences, University of New South Wales, \\ Australian Defence Force Academy, Canberra, ACT 2600, Australia \\ 5 Department of Physics, Astronomy and Geosciences, Towson University, Towson, MD 21252, USA
}

Received 9 March 2017 / Accepted 5 May 2017

\begin{abstract}
Aims. We study the forward shock in the oxygen-rich young supernova remnant (SNR) 1E 0102.2-7219 (1E 0102 in short) via optical coronal emission from [Fe XIV] and [Fe XI]: emission lines that allow for the use of an alternative method to X-rays for this purpose. Methods. We have used the Multi-Unit Spectroscopic Explorer (MUSE) optical integral field spectrograph at the Very Large Telescope (VLT) on Cerro Paranal to obtain deep observations of SNR 1E 0102 in the Small Magellanic Cloud. Our observations cover the entire extent of the remnant with a seeing limited spatial resolution of $0.7^{\prime \prime} \equiv 0.2 \mathrm{pc}$ at the distance of $1 \mathrm{E} 0102$.

Results. Our MUSE observations unambiguously reveal the presence of [Fe XIV] and [Fe XI] emission in 1E 0102. The emission largely arises from a thin, partial ring of filaments surrounding the fast-moving O-rich ejecta in the system. The brightest [Fe XIV] and [Fe XI] emission is found along the eastern and north-western sides of 1E 0102, where shocks are driven into denser ISM material, while fainter emission along the northern edge reveals the location of the forward shock in lower-density gas, possibly the relic stellar wind cavity. Modeling the eastern shocks and the photoionization precursor surrounding 1E 0102, we derive a pre-shock density $n_{\mathrm{H}}=(7.4 \pm 1.5) \mathrm{cm}^{-3}$, and a shock velocity $330 \mathrm{~km} \mathrm{~s}^{-1}<v_{\mathrm{s}}<350 \mathrm{~km} \mathrm{~s}^{-1}$.
\end{abstract}

Key words. shock waves - ISM: supernova remnants - ISM: individual objects: 1E 0102.2-7219 - techniques: imaging spectroscopy

\section{Introduction}

The supernova remnant (SNR) 1E0102.2-7219 (1E0102 in short; Dopita et al. 1981; Tuohy \& Dopita 1983) is located in the Small Magellanic Cloud (SMC). With an age of $(2054 \pm 584) \mathrm{yr}$ (Finkelstein et al. 2006), it belongs to the class of oxygen-rich (O-rich) young SNRs. The characteristic fast-moving O-rich ejecta in this system - the stellar chunks left-over from the SN explosion - form an intricate set of filaments (both spatially and in velocity space; Finkelstein et al. 2006; Vogt \& Dopita 2010). These ejecta are detected at optical wavelengths primarily via the [O III] $\lambda \lambda 4959,5007 \AA$ lines (but see also Blair et al. 2000) following their encounter at a few $1000 \mathrm{~km} \mathrm{~s}^{-1}$ with the reverse shock (Sutherland \& Dopita 1995).

The forward shock wave triggered by the SN explosion, on the other hand, creates a shell of hot X-ray-emitting gas surrounding the SNR as it ploughs through the surrounding medium. This hot gas phase in SNRs is typically studied via $\mathrm{X}$-ray observations, and the case of 1E 0102 is no exception (see e.g., Hughes et al. 2000; Gaetz et al. 2000; Rasmussen et al. 2001; Flanagan et al. 2004). But this hot phase in SNRs can also be detected via coronal emission such as [Fe XIV] $\lambda 5303 \AA$, [Fe XI] $\lambda 7892 \AA$, and [Fe X] $\lambda 6375 \AA$ (Shklovskii 1967). Accessible at visible wavelengths, these forbidden lines enable ground-based observations of the hot phase in SNRs at high spatial resolution. Perhaps more importantly, these coronal lines are also highly temperature sensitive (Kurtz et al. 1972),

\footnotetext{
$\star$ ESO Fellow.
}

and thus allow differentiation of the different structural layers of the forward-shocked medium, otherwise blended at $\mathrm{X}$-ray wavelengths. Forbidden coronal Fe emission has already been detected in several SNRs (Woodgate et al. 1974, 1979; Murdin et al. 1978; Lucke et al. 1979; Dopita \& Mathewson 1979; Itoh 1979). Most recently, Dopita et al. (2016) used integral field spectroscopy to tie the [Fe XIV] emission in SNR N49 to its blast wave, deriving associated shock velocities $v_{\mathrm{s}}=$ (350-400) $\mathrm{km} \mathrm{s}^{-1}$. In this letter, we report the first unambiguous detection and spatial mapping of both [Fe XIV] and [Fe XI] emission from the forward shock and associated denser cloud shocks in SNR 1E 0102.

\section{Observations, data reduction, and post-processing}

SNR 1E 0102 was observed with the Multi-Unit Spectroscopic Explorer (MUSE) in Service Mode at the Very Large Telescope on the night of October 7, 2016, as part of Director Discretionary Time (DDT) program 297.D-5058 (P.I.: F.P.A. Vogt). The observations are comprised of $9 \times 900 \mathrm{~s}$ exposures on-source, interleaved with $180 \mathrm{~s}$ exposures targeting an empty sky field away from the SMC, located at $02^{\mathrm{h}} 07^{\mathrm{m}} 41.0 ;-72^{\circ} 14^{\prime} 43^{\prime \prime}$.0 [J2000]. The large angular separation $\left(\sim 4.9^{\circ}\right)$ between the object field and the sky field led us to create individual observing blocks (OBs) for each exposure, assembled into three OSOSO concatenations. These concatenations were acquired back-to-back over a $\sim 4 \mathrm{~h}$ period, with 1E0102 below 1.6 airmass in all cases and with a 
stable seeing of $0.7^{\prime \prime}$ measured in the SGS throughout. Small spatial shifts $\sim 0.7^{\prime \prime}$ and position angle changes of $90^{\circ}$ were applied to each object exposure to help remove the residual background artefacts associated with the 24 individual integral field spectrographs within MUSE.

Each on-target exposure was reduced individually and later combined into a single cube using the REFLEX (Freudling et al. 2013) MUSE workflow (v1.6). The combined datacube corresponds to $8100 \mathrm{~s}$ on-source, with a spatial full-width at halfmaximum (FWHM) measured on stars in the field-of-view of $0.7^{\prime \prime}$ in the V-band. Spectrally, the cube extends from $4750 \AA$ to $9350 \AA$ in steps of $1.25 \AA$. The combined datacube has a size of $323 \times 326$ spatial pixels (spaxels) $\equiv 64.6^{\prime \prime} \times 65.2^{\prime \prime} \equiv$ $19.4 \mathrm{pc} \times 19.6 \mathrm{pc}$, assuming a distance to the SMC of $62 \mathrm{kpc}$ (Graczyk et al. 2014; Scowcroft et al. 2016). For consistency with the Second Digitized Sky Survey (DSS-2) $R$-band image of the area, the WCS coordinates of the datacube were shifted by +6 spaxels in the $x$-direction and -0.5 spaxels in the $y$-direction. Every spectrum in the datacube was corrected for Galactic extinction along the line of sight using the Schlafly \& Finkbeiner (2011) recalibration of the Schlegel et al. (1998) infrared-based dust map, which assumes a Fitzpatrick (1999) reddening law with $R v=3.1$ and a different source spectrum than Schlegel et al. (1998). This correction was performed using the BRUTUS code (Vogt et al. 2017a) with $A_{\mathrm{B}}=0.134$ and $A_{V}=0.101$ extracted from the NASA Extragalactic Database.

The numerous stars across the MUSE field-of-view coupled to a spatially-varying nebular continuum complicate the extraction and analysis of the emission lines associated with $1 \mathrm{E} 0102$. Similarly to Vogt et al. (2017b), we rely on the Locally Weighted Scatterplot Smoothing algorithm (LOWESS; Cleveland 1979) to individually fit the continuum for each spatial pixel (spaxel) in the datacube. The LOWESS algorithm provides non-parametric fits that are robust against the presence of hot pixels and/or emission lines via an iterative fitting \& outlier rejection approach. However, the spectral width of emission lines associated with the fast-moving O-rich SNR ejecta along certain lines of sight imply that performing a single LOWESS fit is satisfactory for the majority of spaxels - but not all. To ensure a reliable continuum fit for all spaxels, we adopt the following procedure:

1. Perform an initial low-resolution and robust LOWESS fit (using a spectral windowing size of $920 \AA$ ).

2. For each spectrum, crop all spectral pixels brighter than $5 \times$ the intensity level of the initial LOWESS fit. This effectively crops strong emission lines like [O III] $\lambda \lambda 5007,4959 \AA$ which are much brighter than the continuum and affect LOWESS fits with smaller spectral windowing.

3. Perform a second LOWESS fit on the masked spectrum using smaller (sharper) windowing size of $250 \AA$.

4. Interpolate the second LOWESS fit over the masked pixels using Akima splines (Akima 1970).

The efficiency of this procedure is illustrated in Fig. 1, where we compare the MUSE datacube before and after continuum subtraction. The procedure is not perfect, and leads to residual artifacts associated with the brightest stars. For those, our continuum-removal procedure fails to account for the (sometimes numerous) absorption features in their high $\mathrm{S} / \mathrm{N}$ spectra. For our present analysis, these artifacts do not significantly affect the spectral regions around [Fe XIV] $\lambda 5303 \AA$, [Fe XI] $\lambda 7891 \AA$, and [O III] $\lambda \lambda 5007,4959 \AA$ and will thus not be discussed further.
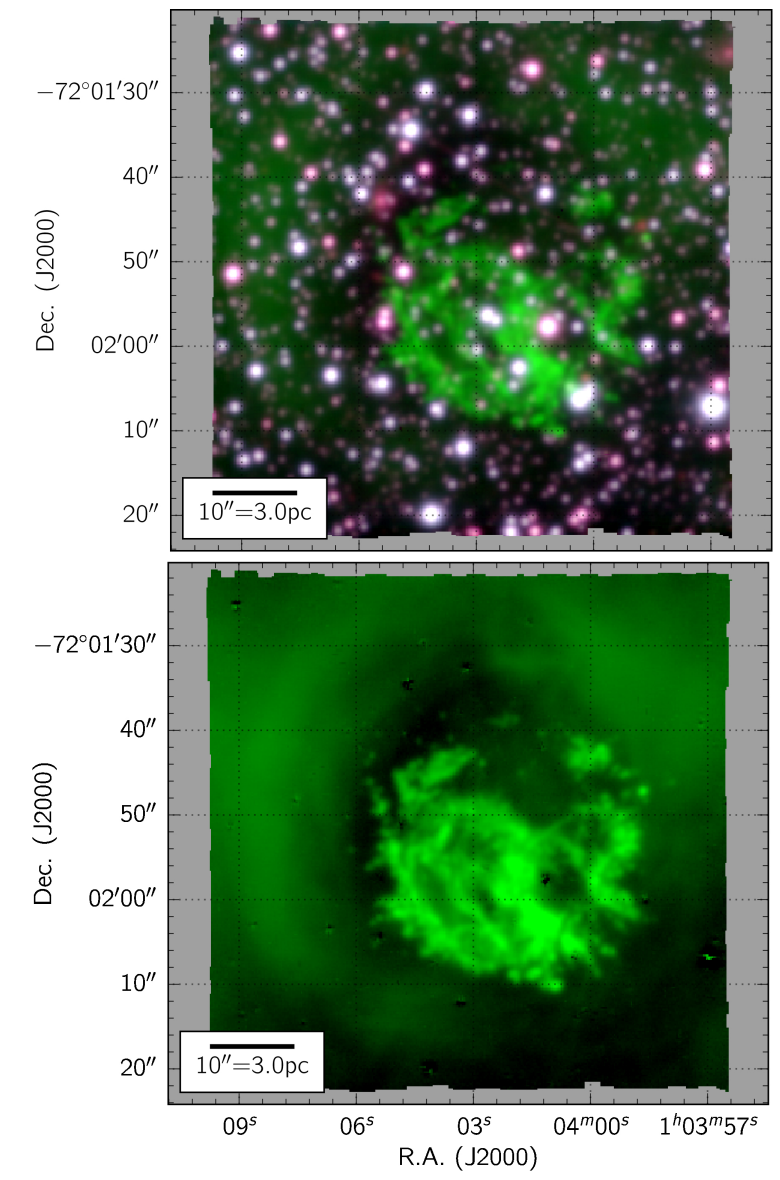

Fig. 1. Pseudo-RGB images constructed from the combined MUSE datacube before (top) and after (bottom) removing the stellar and nebular continuum in each spaxel, with the $R, G$, and $B$ channels corresponding to the summed $6900 \AA \rightarrow 7100 \AA, 4900 \AA \rightarrow 5100 \AA$, and $5300 \AA \rightarrow 5500 \AA$ (observed) spectral ranges, respectively. The intensity stretch of each channel differs between both images for greater clarity. Stars appear in hues of blue and red, whereas the O-rich fast-moving ejecta are visible as bright green filaments.

\section{Results}

We present in Fig. 2 the integrated flux maps for the [Fe XIV] and $[\mathrm{Fe} \mathrm{XI}]$ coronal lines, extracted from our continuum subtracted MUSE datacube. A thin ellipsoidal structure can be seen in these emission line maps, with brighter rims towards the East and the north-west. A faint arc predominantly visible in [Fe XIV] is located west of the SNR center. Towards the south, the coronal emission is fainter but also appears more spatially extended. The [Fe XIV] and [Fe XI] emission encloses most of the O-rich ejecta and is contained (overall) within the X-ray shell of the SNR observed in Chandra ACIS observations ${ }^{1}$ (see Fig. 3). We marginally detect emission from [Fe X] $\lambda 6375 \AA$ co-spatial with [Fe XIV] and [Fe XI], but the proximity of this line (in wavelength) to bright [O I] $\lambda 6300 \AA$ emission from (redshifted) Orich ejecta significantly complicates its analysis. We delay the discussion of $[\mathrm{Fe} \mathrm{X}]$ emission until careful identification of all the fast-moving ejecta in the datacube (outside the scope of this Letter) has been performed.

1 The Chandra ACIS data was downloaded from http://chandra. harvard.edu/photo/openFITS/xray_data.html 

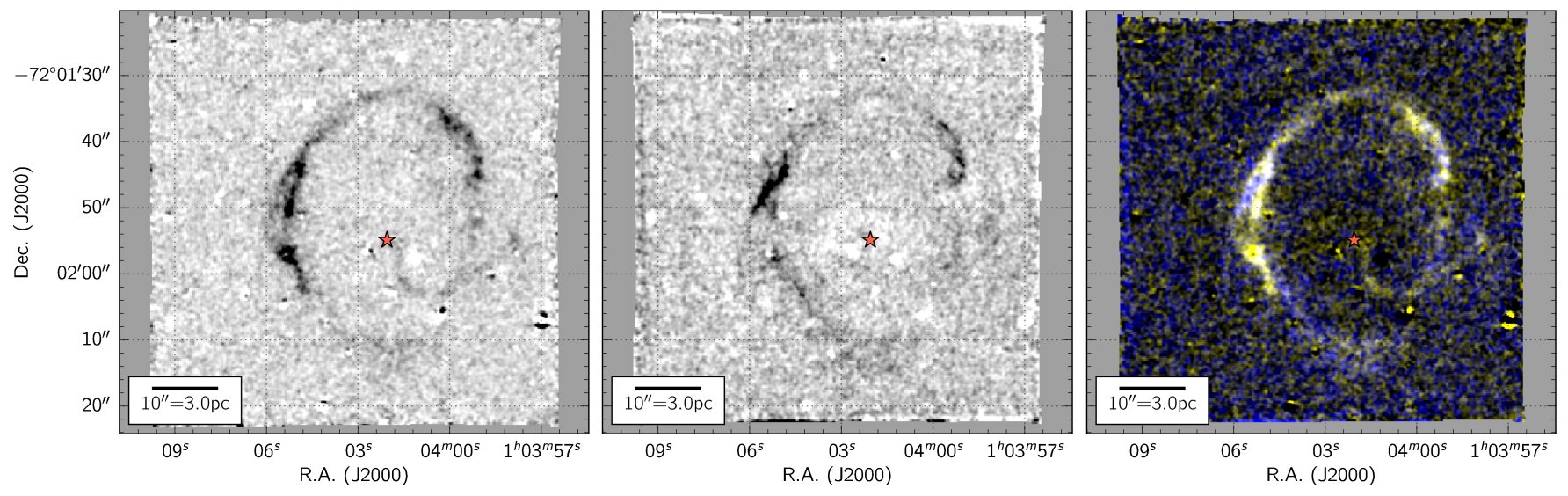

Fig. 2. Left: integrated [Fe XIV] emission. The image was smoothed using a Gaussian kernel with a smoothing scale of 1 spaxel across to enhance the fainter structures. The red star marks the proper motion center of the O-rich ejecta derived by Finkelstein et al. (2006). Middle: idem, but for [Fe XI]. Right: combination of both [Fe XIV] (yellow) and [Fe XI] (blue). North is at the top and east to the left.

\subsection{Surface brightness of the coronal lines}

In the bright [Fe XIV] and [Fe XI] filament on the east side of the SNR, we measure an observed surface brightness $S_{[\mathrm{FexvI}] \mathrm{obs}}=$ $(1.4 \pm 0.2) \times 10^{-17} \mathrm{erg} \mathrm{cm}^{-2} \operatorname{arcsec}^{-2} \mathrm{~s}^{-1}$ and $S_{[\mathrm{FeXI}], \mathrm{obs}}=$ $(9 \pm 4) \times 10^{-18} \mathrm{erg} \mathrm{cm} \mathrm{cm}^{-2} \operatorname{arcsec}^{-2} \mathrm{~s}^{-1}$. At this location, the projected outer radius of the coronal line-emitting region is about 14.3 arcsec or $4.3 \mathrm{pc}$. The projected width of the filament is $\sim 0.5 \mathrm{pc}$, which for a spherical shell implies that the flux adds over a path length through the filament of $4.4 \mathrm{pc}$. The observed surface brightness of the shell is thus enhanced via limb-brightening by a factor $\eta \cong 9$. Using an additional reddening correction of $E(B-V)=0.04$ to account for the SMC (following Blair et al. 2000), implying a line flux attenuation $A_{[\mathrm{Fe} \mathrm{XVI}]}=1.19$ and $A_{[\mathrm{Fe} \mathrm{XI}]}=1.12$ according to the turbulent dust screen model from Fischera \& Dopita (2005), we thus derive the intrinsic surface brightness of the filament $S_{\lambda}=S_{\lambda \text {,obs }} \eta^{-1} A_{\lambda}$ to be: $S_{[\mathrm{FexvI}}=(1.9 \pm 0.3) \times 10^{-18} \mathrm{erg} \mathrm{cm}^{-2} \operatorname{arcsec}^{-2} \mathrm{~s}^{-1}$ and $S_{[\mathrm{Fe} \mathrm{XI}]}=(1.1 \pm 0.5) \times 10^{-18} \mathrm{erg} \mathrm{cm}^{-2} \operatorname{arcsec}^{-2} \mathrm{~s}^{-1}$.

\subsection{Inferred pre-shock density from the precursor emission}

A photoionisation precursor is very evident as a shell of diffuse [O III] emission in Fig. 1. This shell, nearly 2 pc thick, is produced by the strong radiation field of the shocked O-rich ejecta in 1E 0102. From our MUSE data cube and previous observations with the WiFeS integral field spectrograph (Vogt et al. $2017 \mathrm{~b}$ ), we measure a mean $\mathrm{H} \beta$ surface brightness in the northern precursor region (away from the obvious H II regions) of $S_{\mathrm{H} \beta}=(3.3 \pm 1.0) \times 10^{-16} \mathrm{erg} \mathrm{cm}^{-2} \operatorname{arcsec}^{-2} \mathrm{~s}^{-1}$. The inner boundary of the precursor is tightly defined by the size of the [Fe XIV] shell (see Fig. 3): 35 arcsec, or a radius of 5.1 pc. The precursor's outer boundary is, on the other hand, not so well defined. If taken from the edge of the brightening in the [O III] shell, we estimate it to have a diameter of $\sim 52$ arcsec, corresponding to a radius of $\sim 7.8 \mathrm{pc}$. These figures imply a reddening-corrected total $\mathrm{H} \beta$ flux for the precursor of $F_{\mathrm{H} \beta}=(6.4 \pm 2) \times 10^{-13} \mathrm{erg} \mathrm{cm}^{-2} \mathrm{~s}^{-1}$, which, correcting for the distance to the SMC, yields an absolute $\mathrm{H} \beta$ luminosity for the whole precursor (treated as a complete shell $)$ of $L_{\mathrm{H} \beta}=(2.4 \pm 0.8) \times 10^{35} \mathrm{ergs} \mathrm{s}^{-1}$.

We can now estimate the mean density in the [O III]-emitting region around 1E 0102 . The luminosity in $\mathrm{H} \beta$ is $L_{\mathrm{H} \beta}=\epsilon_{\mathrm{H} \beta} V n_{\mathrm{H}}^{2}$, where $\epsilon_{\mathrm{H} \beta}=1.014 \times 10^{-25} \mathrm{erg} \mathrm{cm}^{3} \mathrm{~s}^{-1}$ for Case B hydrogen emissivity (Dopita \& Sutherland 2003, Table B.5, p. 381), $V$ is

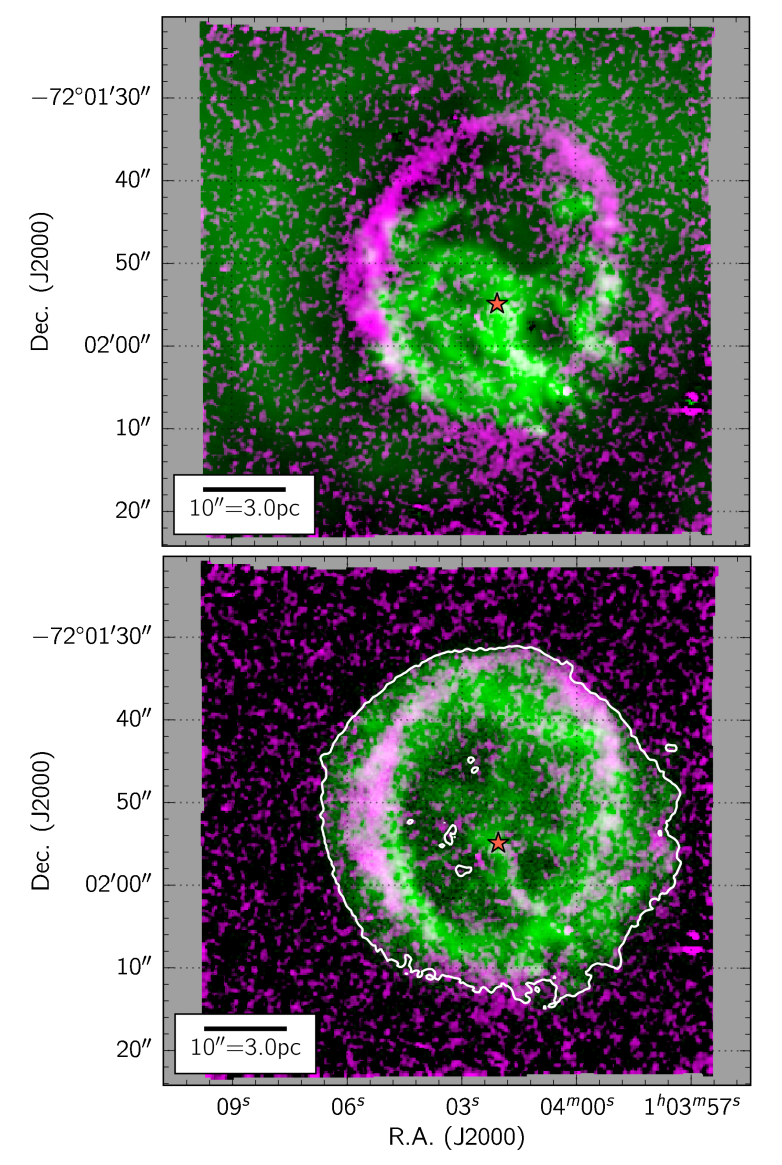

Fig. 3. Top: comparison between the integrated [Fe XIV] emission (purple) and integrated [O III] $\lambda \lambda 4959,5007 \AA$ emission (green). The red star marks the proper motion center of the O-rich ejecta derived by Finkelstein et al. (2006). Bottom: idem, but with the Chandra ACIS $1.1 \rightarrow 2 \mathrm{keV}$ X-ray flux instead (ACIS image credits: NASA/CXC/MIT/D. Dewey et al., and NASA/CXC/SAO/J. DePasquale). The full extent of the X-ray shell is traced with a white contour.

the ionized volume, and $n_{\mathrm{H}}$ is the hydrogen density $\left(\mathrm{cm}^{-3}\right)$. The resulting mean density in the precursor region from this calculation is $n_{\mathrm{H}}=(7.4 \pm 1.5) \mathrm{cm}^{-3}$. 


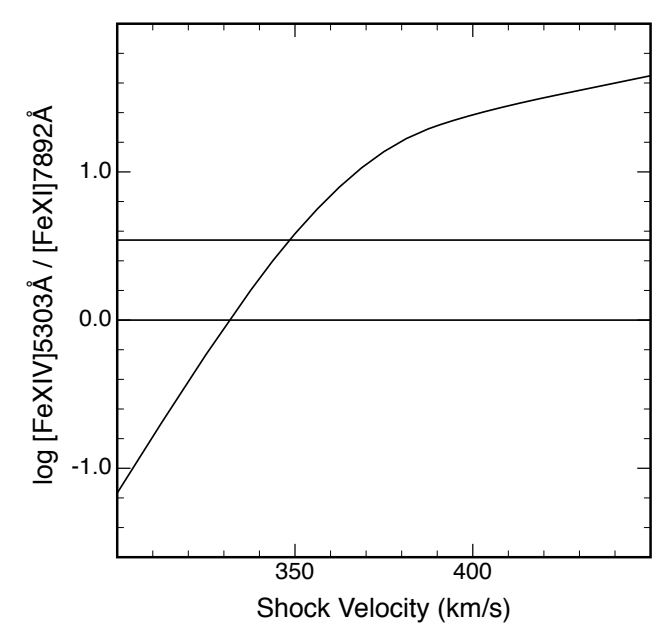

Fig. 4. The computed [Fe XIV] $5303 \AA /[\mathrm{Fe} \mathrm{XI}] 7890 \AA$ ratio as a function of the blast wave velocity. The photometry of the filaments provides observed limits between the two horizontal lines, implying a blast-wave velocity of (330-350) $\mathrm{km} \mathrm{s}^{-1}$.

\subsection{Modeling the blast wave}

We have used the MAPPINGS $\mathrm{v}$ code v10.3 to model the Fe forbidden emission line strengths and ratios for the bright filament on the eastern side of 1E0102. We used the SMC abundances from Russell \& Dopita (1992), with the heavy elements depleted onto dust according to the Jenkins (2009) scheme. The base depletion factor was $\log D_{\mathrm{Fe}}=-1.00$, which is appropriate for the diffuse ionized ISM. Our shock models have a reference preshock hydrogen density $n_{\mathrm{H}}=10 \mathrm{~cm}^{-3}$ and are run for $2000 \mathrm{yr}$ : long enough for the ionization time-scale of the relevant $\mathrm{Fe}$ species to be short in comparison, so that we settle into a steadystate solution.

We find that the predicted flux ratio [Fe XIV] $\lambda 5303 \AA$ over [Fe XI] $\lambda 7892 \AA$ is very sensitive to the shock velocity and so provides an accurate way of determining this parameter (see Fig. 4). The observations put limits on this flux ratio of between 1.0 and 3.7, which therefore implies that the shock velocity of the eastern filament is in the range $330 \mathrm{~km} \mathrm{~s}^{-1}<v_{\mathrm{s}}<350 \mathrm{~km} \mathrm{~s}^{-1}$. For the model with $v_{s}=340 \mathrm{~km} \mathrm{~s}^{-1}$, we infer that $S_{[\mathrm{FexI}]}=$ $3.9 \times 10^{-18}\left(n_{10}\right)\left(D_{\mathrm{Fe}} / 0.1\right) \mathrm{erg} \mathrm{cm}{ }^{-2} \operatorname{arcsec}^{-2} \mathrm{~s}^{-1}$, where $n_{10}$ is the assumed pre-shock hydrogen density in units of $10 \mathrm{~cm}^{-3}$ and $D_{\mathrm{Fe}}$ is the assumed depletion factor of Fe in the ISM. Likewise, $S_{\text {[Fe XI] }}=3.3 \times 10^{-18}\left(n_{10}\right)\left(D_{\mathrm{Fe}} / 0.1\right) \mathrm{erg} \mathrm{cm}^{-2} \operatorname{arcsec}^{-2} \mathrm{~s}^{-1}$. Comparing these numbers with those in Sect. 3.1, we infer that the pre-shock density for the eastern filament is $n_{\mathrm{H}}=3-5 \mathrm{~cm}^{-3}$; in reasonable agreement with the density estimated from the precursor luminosity above of $n_{\mathrm{H}}=(7.4 \pm 1.5) \mathrm{cm}^{-3}$.

The picture emerging from our analysis is that of a supernova remnant expanding into an asymmetric bubble swept out by the progenitor WR star winds. The bubble is narrower along the east-west direction (as noted by Finkelstein et al. 2006), and the blast wave of 1E0102 has struck denser gas filaments on these sides of the remnant. In doing so, the main shock wave moving outwards at a few $1000 \mathrm{~km} \mathrm{~s}^{-1}$ in a low density medium with $n_{\mathrm{H}} \sim 0.1 \mathrm{~cm}^{-3}$, has generated the local, slower, non-radiative shocks with speeds of $\sim 340 \mathrm{~km} \mathrm{~s}^{-1}$ simulated above. Along the northern and southern edges, the SNR is still expanding through the wind cavity (traced by the dark [O III] hole seen in Fig. 3). In particular, the northern edge of the X-ray remnant fills most, but not all of the [O III] hole; a fact consistent with the propagation of the blast wave through a low-density cavity.
Acknowledgements. We thank the anonymous referee for a prompt and constructive report. This research has made use of BRUTUS, a Python module to process data cubes from integral field spectrographs hosted at http://fpavogt.github.io/brutus/. For this analysis, BRUTUS relied on STATSMODEL (Seabold \& Perktold 2010), MATPLOTLIB (Hunter 2007), ASTROPY, a community-developed core Python package for Astronomy (Astropy Collaboration et al. 2013), APLPY, an open-source plotting package for Python hosted at http://aplpy.github.com, and MONTAGE, funded by the National Science Foundation under Grant Number ACI-1440620 and previously funded by the National Aeronautics and Space Administration's Earth Science Technology Office, Computation Technologies Project, under Cooperative Agreement Number NCC5-626 between NASA and the California Institute of Technology. This research has also made use of the ALADIN interactive sky atlas (Bonnarel et al. 2000), of SAOIMAGE DS9 (Joye \& Mandel 2003) developed by Smithsonian Astrophysical Observatory, of NASA's Astrophysics Data System, and of the NASA/IPAC Extragalactic Database (NED; Helou et al. 1991) which is operated by the Jet Propulsion Laboratory, California Institute of Technology, under contract with the National Aeronautics and Space Administration. IRS was supported by Australian Research Council Laureate Grant FL0992131. PG thanks the Stromlo Distinguished Visitor Programme. FPAV and IRS thank the CAASTRO AI travel grant for generous support. We also thank Ashley Ruiter for helpful comments on the manuscript. Based on observations made with ESO Telescopes at the La Silla Paranal Observatory under programme ID 297.D-5058[A].

\section{References}

Akima, H. 1970, J. ACM, 17, 589

Astropy Collaboration, Robitaille, T. P., Tollerud, E. J., et al. 2013, A\&A, 558, A33

Blair, W. P., Morse, J. A., Raymond, J. C., et al. 2000, ApJ, 537, 667

Bonnarel, F., Fernique, P., Bienaymé, O., et al. 2000, A\&AS, 143, 33

Cleveland, W. S. 1979, J. Am. Stat. Assoc., 74, 829

Dopita, M. A., \& Mathewson, D. S. 1979, ApJ, 231, L147

Dopita, M. A., \& Sutherland, R. S. 2003, Astrophysics of the Diffuse Universe (Berlin, Heidelberg, New York: Springer)

Dopita, M. A., Tuohy, I. R., \& Mathewson, D. S. 1981, ApJ, 248, L105

Dopita, M. A., Kewley, L. J., Sutherland, R. S., \& Nicholls, D. C. 2016, Ap\&SS, 361,61

Finkelstein, S. L., Morse, J. A., Green, J. C., et al. 2006, ApJ, 641, 919

Fischera, J., \& Dopita, M. 2005, ApJ, 619, 340

Fitzpatrick, E. L. 1999, PASP, 111, 63

Flanagan, K. A., Canizares, C. R., Dewey, D., et al. 2004, ApJ, 605, 230 Freudling, W., Romaniello, M., Bramich, D. M., et al. 2013, A\&A, 559, A96

Gaetz, T. J., Butt, Y. M., Edgar, R. J., et al. 2000, ApJ, 534, L47

Graczyk, D., Pietrzyński, G., Thompson, I. B., et al. 2014, ApJ, 780, 59

Helou, G., Madore, B. F., Schmitz, M., et al. 1991, Databases and On-line Data in Astronomy, 171, 89

Hughes, J. P., Rakowski, C. E., \& Decourchelle, A. 2000, ApJ, 543, L61

Hunter, J. D. 2007, Comput. Sci. Eng., 9, 90

Itoh, H. 1979, Nature, 281, 656

Jenkins, E. B. 2009, ApJ, 700, 1299

Joye, W. A., \& Mandel, E. 2003, in Astronomical Data Analysis Software and Systems XII, ASP Conf. Ser., 295, 489

Kurtz, D. W., Vanden Bout, P. A., \& Angel, J. R. P. 1972, ApJ, 178, 701

Lucke, R. L., Woodgate, B. E., Culhane, J. L., Socker, D. G., \& Zarnecki, J. C. 1979, ApJ, 228, 763

Murdin, P., Clark, D. H., \& Culhane, J. L. 1978, MNRAS, 183, 79

Rasmussen, A. P., Behar, E., Kahn, S. M., den Herder, J. W., \& van der Heyden, K. 2001, A\&A, 365, L231

Russell, S. C., \& Dopita, M. A. 1992, ApJ, 384, 508

Schlafly, E. F., \& Finkbeiner, D. P. 2011, ApJ, 737, 103

Schlegel, D. J., Finkbeiner, D. P., \& Davis, M. 1998, ApJ, 500, 525

Scowcroft, V., Freedman, W. L., Madore, B. F., et al. 2016, ApJ, 816, 49

Seabold, S., \& Perktold, J. 2010, in Proc. of the 9th Python in Science Conference, 57

Shklovskii, I. S. 1967, Sov. Astron., 10, 594

Sutherland, R. S., \& Dopita, M. A. 1995, ApJ, 439, 381

Tuohy, I. R., \& Dopita, M. A. 1983, ApJ, 268, L11

Vogt, F., \& Dopita, M. A. 2010, ApJ, 721, 597

Vogt, F. P. A., Pérez, E., Dopita, M. A., Verdes-Montenegro, L., \& Borthakur, S. 2017a, A\&A, 601, A61

Vogt, F. P. A., Seitenzahl, I. R., Dopita, M. A., \& Ruiter, A. J. 2017b, PASP, 129, 058012

Woodgate, B. E., Stockman, Jr., H. S., Angel, J. R. P., \& Kirshner, R. P. 1974, ApJ, 188, L79

Woodgate, B. E., Lucke, R. L., \& Socker, D. G. 1979, ApJ, 229, L119 